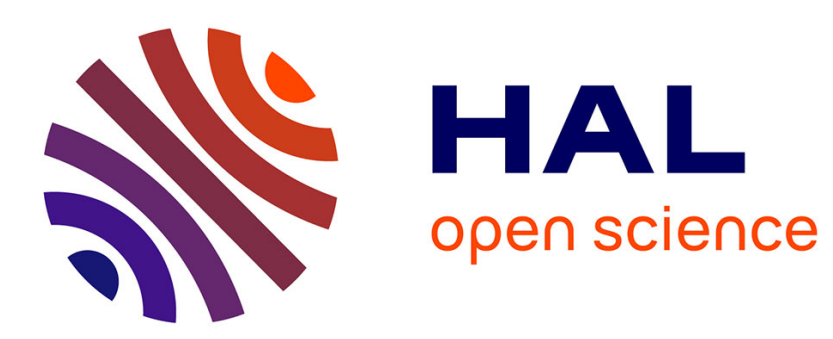

\title{
Periodic networks of disclination lines: application to metal structures
}

\author{
J.F. Sadoc
}

\section{To cite this version:}

J.F. Sadoc. Periodic networks of disclination lines: application to metal structures. Journal de Physique Lettres, 1983, 44 (17), pp.707-715. 10.1051/jphyslet:019830044017070700 . jpa-00232254

\section{HAL Id: jpa-00232254 https://hal.science/jpa-00232254}

Submitted on 1 Jan 1983

HAL is a multi-disciplinary open access archive for the deposit and dissemination of scientific research documents, whether they are published or not. The documents may come from teaching and research institutions in France or abroad, or from public or private research centers.
L'archive ouverte pluridisciplinaire HAL, est destinée au dépôt et à la diffusion de documents scientifiques de niveau recherche, publiés ou non, émanant des établissements d'enseignement et de recherche français ou étrangers, des laboratoires publics ou privés. 
Classification

Physics Abstracts

$61.00-61.70$

\title{
Periodic networks of disclination lines : application to metal structures
}

\author{
J. F. Sadoc \\ Laboratoire de Physique des Solides, Université Paris-Sud, 91405 Orsay, France
}

(Reçu le 17 février 1983, révisé le 21 avril, accepté le 4 juillet 1983)

\begin{abstract}
Résumé. - Nous décrivons des structures complexes pour quelques métaux (Mn, W, U etc...) et montrons comment elles peuvent être définies comme des structures polytétraédriques réticulées par des lignes de disinclinaisons périodiques.
\end{abstract}

Abstract. - Complex structures for some metals (Mn, W, U etc...) are described. It is shown how it is possible to define these structures as polytetrahedral structures reticulated by a periodic network of disclination lines.

\section{Introduction.}

Recent developments of the study of random close packed structures are based on the use of a curved space in which a regular structure is used as reference state for the order in the amorphous state [1]. Using the hypothesis that all interstices formed by spherical atoms have a tetrahedral symmetry, the reference order is realized by a $\{3,3,5\}$ polytope [2].

This geometrical figure can be described on a 3-sphere embedded in a 4D Euclidean space. This figure is regular in that sense that all vertices are surrounded by a regular icosahedron.

For example on figure 1 we observe a lattice which is a tiling of the $2 \mathrm{D}$ plane by pentagons. The coordination number at vertices is 3 or 4 . A disclination procedure can convert a dodecahedron to this lattice; in this case disclination points correspond to the 4-fold coordinated vertices. An accurate disclination procedure in $2 \mathrm{D}$ is described in reference 3 . Notice that the defect-free structure of reference is not a lattice in the Euclidean plane, but a polyhedron defined on a 2-sphere.

The description of realistic structures needs a mapping of the curved space onto Euclidean space. That can be achieved by a disclination procedure as disclination lines are known to change the curvature of the space. In $2 \mathrm{D}$ cases it has been shown that non-crystalline $2 \mathrm{D}$ structures can be obtained if disclination points (in 2D) are non periodic, but if disclination points are arranged to form a lattice, then a periodic structure is obtained.

The aim of the present paper is to answer this question : are there periodic structures in dense metal which can be described using the concept of an icosahedral order (with a reference to the $\{3,3,5\}$ polytope) associated with disclination lines periodically arranged ? 


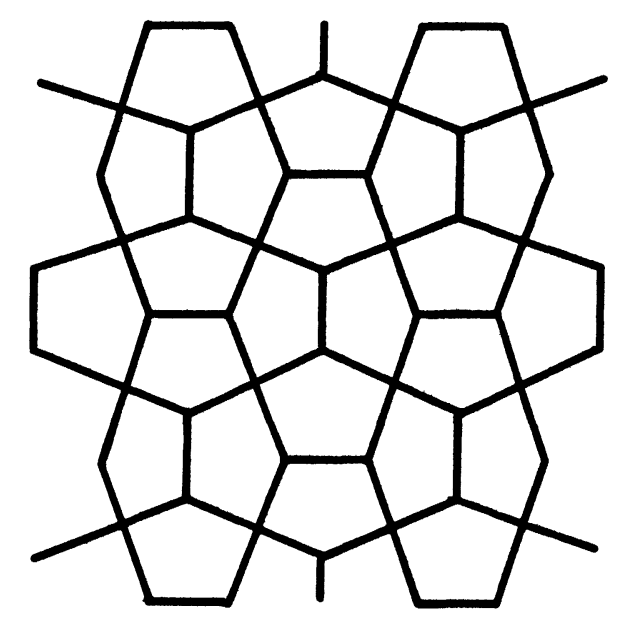

Fig. 1. - Tiling of the plane with pentagons. This network can be generated out of a dodecahedron using a disclination procedure. Disclination points appear on 4-fold coordinated vertices.

\section{2. $\{3,3,5\}$ Polytope and disclination lines.}

In $2 \mathrm{D}$ the dodecahedron is the unique way to realize a tiling of a surface with regular pentagons, keeping a 3-fold coordination for vertices. In $3 D$ the $\{3,3,5\}$ polytope is the way to realize a tiling of 3D space by tetrahedra, keeping a 12-fold coordination for vertices. But in the first example the surface is curved like a 2 -sphere, and in the $\{3,3,5\}$ example the space is curved like a 3-sphere.

In these structures disclinations change the curvature and a given density of disclination can reduce the curvature to zero. The $\{3,3,5\}$ polytope can be described with the help of the spherical torus [4]. The spherical torus is a surface (2D) which lies in the 3D spherical space. Like a classical torus it can be built from a cylinder closed on itself, but due to the $3 \mathrm{D}$ space curvature there is not any distortion during this operation. So the spherical torus is a developable surface. There are two useful lines to define this surface : the " axes " of this torus. In a classical torus one of these axes is a straight line, the other one is a circle. In spherical space the two axes are great circles of the 3 -sphere.

The spherical torus divides the 3-sphere into two identical half spherical spaces. 100 vertices of the $\{3,3,5\}$ polytope are on two tori parallel to the spherical torus. These two toric layers have the same axes as the spherical torus. They can be obtained by identification of opposite bases of two cylinders having the same axes as the cylinder used to define the spherical torus. One of these layers is drawn on figure 2.

There are also 10 vertices on each axis of the torus. So are the 120 vertices of the $\{3,3,5\}$ polytope. Each of these vertices has a coordination polyhedron which is an icosahedron. For instance that can be observed for one of the vertices lying on an axis : a pentagonal anti-prism extracted from the toric layer contains 10 of the vertices of the coordination icosahedron, the 2 other vertices being on the axis.

Now we suppose a disclination line running on an axis of the torus. This defect change pentagonal anti-prism into hexagonal anti-prism and consequently the coordination polyhedra of vertices lying on the disclination line are 14-polyhedra. The defect also adds new vertices in the whole structure but the local topology around these new vertices is identical to the topology of the original vertices. A disclination can be added on the other torus axis in order to have a most symmetric procedure. In this case the new polytope contains 24 vertices which are 14-fold coor- 


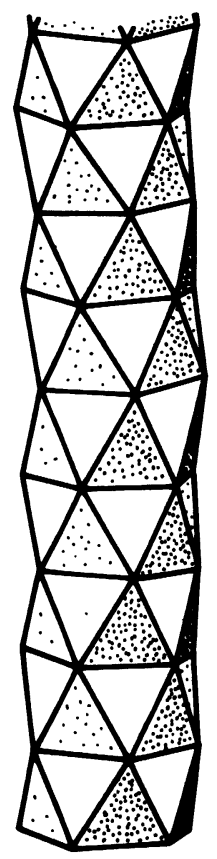

Fig. 2. - One of the two toric layers appearing in the $\{3,3,5\}$ polytope. The two ends have to be identified in order to get a torus. This configuration can be obtained by piling up pentagonal anti-prisms.

dinated and 144 vertices which are 12-fold coordinated and undistinguishable from those of the original $\{3,3,5\}$. A new disclination procedure can be done in a similar way and therefore the curvature is decreased by iteration.

The structures described in the next paragraph are supposed to be the final state after an infinite number of iterations. This hypothesis is based on a local argument : disclination lines change the topology only in their neighbourhood; all other vertices (original in the $\{3,3,5\}$ reference structure or added by disclination) have a local coordination which is unchanged.

\section{Some surprising structures.}

In this paper, only pure metal structures are described (with an exception for the Laves phases). But all the intermetallic alloys in which there are some atoms with an icosahedral coordination can be studied from this point of view [5].

3.1 The $\beta$-TUNGSTEN STRUCTURE [4]. - The A15 structure is probably the simplest one. It is observed for numerous alloys but also for pure tungsten in the $\mathrm{W}_{\beta}$ form. Schematically we can use the formula $\mathrm{W}_{3}^{\mathrm{a}} \mathrm{W}^{\mathrm{b}}$ for this pure metal in which there are two types of atomic positions : $\mathrm{W}^{\mathrm{a}}$ and $\mathrm{W}^{\mathbf{b}}$. This structure is also observed for $\mathrm{Nb}$ [7] and $\mathrm{Cr}[8,9]$.

The elementary cell of the structure is a cube. An icosahedron with three orthogonal $A_{2}$ symmetry axes superposed to the three $\mathrm{A}_{4}$ axes of the cube is inscribed in the cubic cell. So, 12 atoms are on the faces of the cube, a 13th one is in the centre of the cube. There are also 8 atoms on each corner of the cube. So there are 8 atoms in the cell : 2 of them are $\mathrm{W}^{\mathrm{b}}$ with an icosahedral coordination, and 6 of them are $W^{a}$ with a coordination polyhedron having 14 vertices.

Looking at this 14 vertex polyhedron (Fig. 3) it is clear that it can be obtained by a disclination (Fig. 4) (rotation dislocation) going through an $\mathrm{A}_{5}$ axis of an icosahedron. In an infinite structure, 

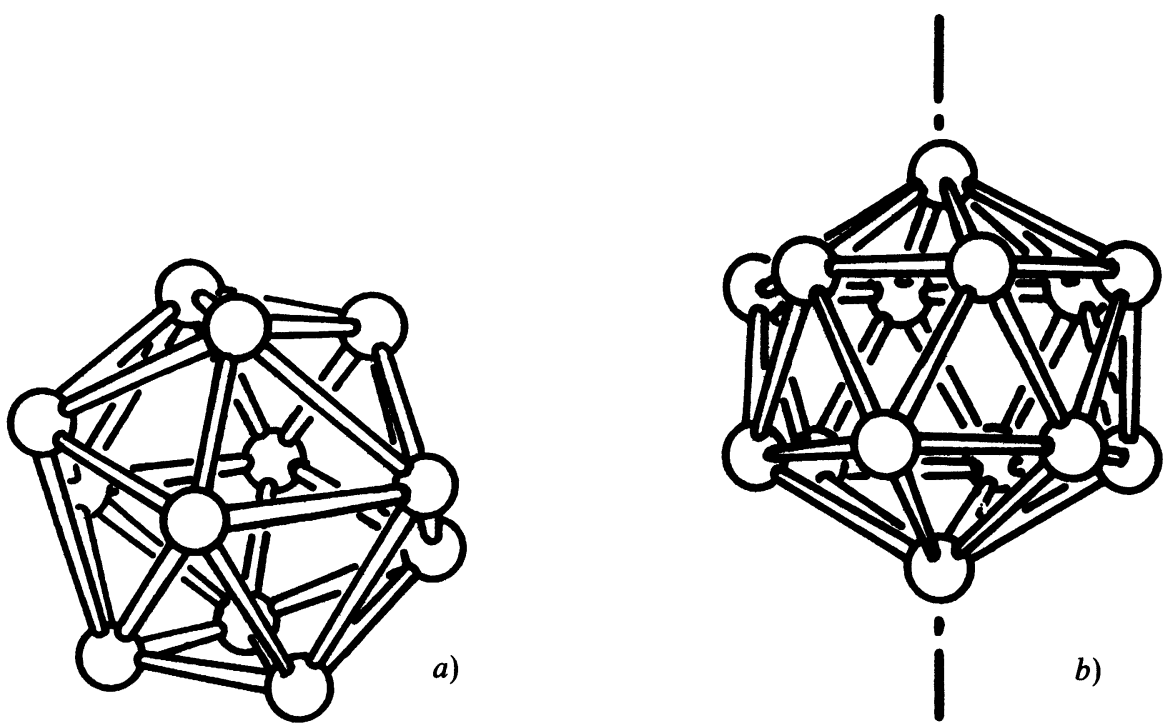

Fig. 3. - The coordination polyhedra in $\beta$-tungsten $: a$ ) for atom $\mathrm{W}^{\mathrm{b}}$ (coordination number $=12$ ), b) for atom $W^{\mathbf{a}}$ (coordination number $=14$ ).
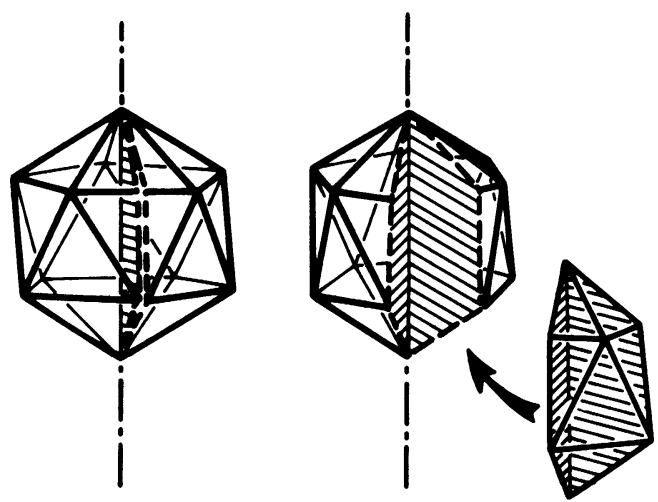

Fig. 4. - Disclination procedure changing an icosahedron into a 14-vertex coordination polyhedron.

a disclination line must have no ends. Taking into account the situation of the $\mathrm{W}^{\mathrm{a}}$ atoms we observe that straight lines running in the faces of cubic cells can be, with this description, defined as disclination lines (Fig. 5). These disclination lines can also be defined in term of the Voronoi cell description. The Voronoi cells for $\mathrm{W}^{\mathrm{b}}$ atoms are dodecahedra with 12 pentagonal faces. The Voronoï cells for $\mathbf{W}^{\mathbf{a}}$ atoms are polyhedra with 2 hexagonal faces (in opposite position) and 12 pentagonal faces. The disclination is a line going through the 2 hexagonal faces. The disclination operation change 2 opposite pentagons of a dodecahedron into two hexagons and add 2 new pentagons on the polyhedron. In this case the characteristic angle of the disclination is $2 \pi / 5$. In a topological sense we can say that the $W_{\beta}$ structure is a $\{3,3,5\}$ polytope with atoms on vertices, in which disclinations lines have changed the curvature and the coordination polyhedra of three out of four atoms. 


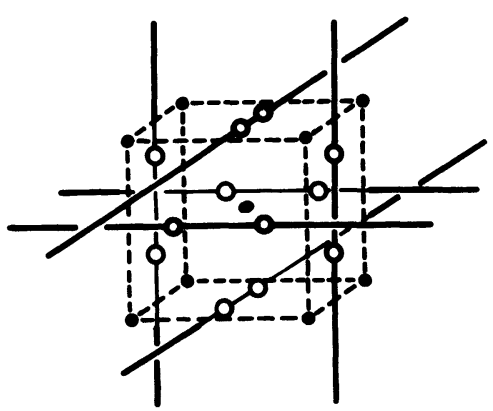

Fig. 5. $-\beta$-tungsten unit cell and the disclination lines.

Even if this description does not seem useful to describe $W_{\beta}$ it is of great interest in order to understand the rôle of disclinations in ordered and disordered structure. Perhaps also the important structural unit in dense structure is, more than the icosahedron, the pentagonal bipyramid obtained by packing five tetrahedra with a common edge. The relative number of these pentagonal bipyramids changed into hexagonal bipyramids by disclinations is only $6 / 54=1 / 9$.

3.2 The Laves Phases Structure $\left(\mathrm{Cu}_{2} \mathrm{Mg}\right)$. - The Laves phase is also an example of structure which can be described as a $\{3,3,5\}$ polytope with disclination lines lowering the curvature to zero. But in this case disclination lines intersect mutually, building a simple network. For this reason we present this example nevertheless it is not a structure for pure metal.

Laves phases are described with cubic cell containing 8 atoms of type A (atom with a large Goldsmith radius) arranged like carbon atoms in a diamond structure. There are 16 atoms of type B filling the free space of a diamond structure. The coordination polyhedron for a B atom is a slightly distorted icosahedron with $6 \mathrm{~B}$ atoms and $6 \mathrm{~A}$ atoms on its vertices. The coordination polyhedron for an $\mathrm{A}$ atom has 16 vertices, which are $4 \mathrm{~A}$ atoms and $12 \mathrm{~B}$ atoms. This 16 vertex polyhedron can be obtained by a disclination procedure from an icosahedron. The study of the Voronoï cell is helpful to observe the configuration of the disclination lines. The Voronoi cell of the $\mathrm{A}$ atoms is a polyhedron with 16 faces. 4 faces are hexagons, 12 other ones are pentagons. So we can consider that 4 half disclination lines are going in the centre of the polyhedron through the hexagonal faces. That can also be described as 2 disclinations intersecting each other on the centre, and leading to six angles of $109^{\circ} 28^{\prime}$.

Now we can describe this structure as a $\{3,3,5\}$ polytope in which disclinations change the curvature. These disclination lines are interconnected exactly the same way as bonds in a diamond structure. They are going straight from $\mathrm{A}$ atom to $\mathrm{A}$ atom and form angles on all the $\mathrm{A}$ atoms. So we define a periodic network of intersecting disclination lines. In this structure the change of the coordination number for atoms located on a disclination line is greater than in the $\mathbf{W}_{\beta}$ case. But the relative number of unperturbed coordination polyhedra is $2 / 3$. The relative number of pentagonal bipyramids changed into hexagonal bipyramids is in this case $2 / 20=1 / 10$. This ratio is close to the value obtained in $W_{\beta}(1 / 9)$. The small variation is probably related to the angle of $109^{\circ} 28^{\prime}$ done by disclination lines on the $A$ sites.

3.3 THE $\alpha$-MANGANESE AND THE $\beta$-URANIUM STRUCTURES. - In these two examples of pure metal the structure is extremely complex. There are 58 manganese atoms in a big cubic cell. The $U_{\beta}$ cell is tetragonal and contains 30 atoms. There are 4 different types of atomic position in the $\mathrm{Mn}_{\alpha}[8]$ structure and 5 different types in the $\mathrm{U}_{\beta}$ structure [9]. The different coordination polyhedra of the $\mathrm{Mn}_{\alpha}$ structure are represented in figure 6.

It can be observed that there is a failure when the type III and the type IV polyhedra are joined together. On the figure $6 a$ which presents the type IV coordination polyhedron one can observe 
b)

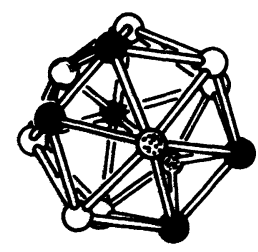

a)

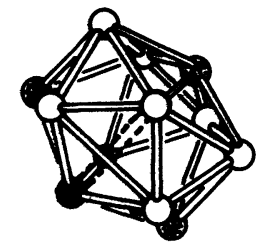

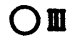

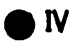

c)

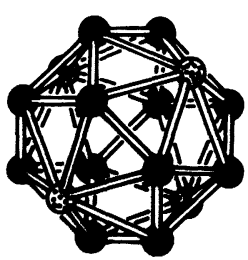

OI

d)

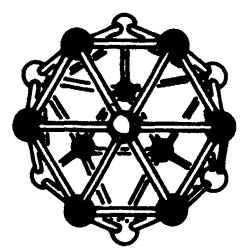

(중 II

Fig. 6. - The coordination polyhedra in $\alpha$-manganese : $a$ ) for type IV atoms (coordination number $=12$ ), b) for type III atoms (coordination number =14), c) for type I atoms (coordination number $=16$ ), d) for type II atoms (coordination number $=16$ ).

on the front face a distance (which is represented by a link) between two type III atoms. But this distance does not appear on the type III coordination polyhedron (Fig. 6b). It is replaced by the dashed link of the figure $6 a$.

The choice of two coherent coordination polyhedra lead to the insertion of a dipole of disclination lines (positive and negative disclination).

In a first approximation the $\mathrm{Mn}_{\alpha}$ structure can be described as $\{3,3,5\}$ polytope containing a periodic network of disclination lines. It is formed by two interpenetrating networks with nodes crossed by one disclination line (coordination $=14$ ) and nodes crossed by 2 disclination lines (coordination $=16$ ).

In a second step, using two coherent coordination polyhedra new disclination lines have to be introduced in pairs. This new disclination pair is needed to allow the bending of the disclination line going through the type III atoms. The disclination dipole acts like a dislocation allowing bending of disclinations.

Coordination polyhedra of the $\mathrm{U}_{\beta}$ are similar to those which are observed in $\mathrm{W}_{\beta}$ and Laves phase structure except for a 15 vertex polyhedron (Fig. 7). It can be described as obtained from an icosahedron by 3 coplanar disclination half-lines intersecting on the centre. The $U_{\beta}$ disclination network (Fig. 8) is formed by two types of disclination lines. One family consists of parallel lines going through points with the coordination number equal to 14 . Disclinations of the other family remain in parallel planes and form a 3-coordinated network.

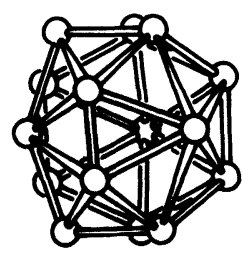

Fig. 7. - The coordination polyhedra in $\beta$-uranium. There are 5 different types of atoms. Coordination polyhedra are icosahedra (like Fig. 1a) for type I and type IV atoms. Coordination polyhedra are 14-vertex polyhedra (like Fig. 1b) for type III and V atoms. In the figure is shown the 15-vertex coordination polyhedron for type II atoms. 


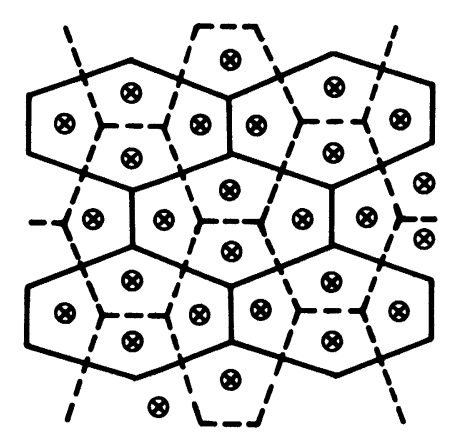

Fig. 8. - The $\beta$-uranium disclination network. There are two types of alternate planes containing disclination lines. The network of disclinations is drawn in full line for one plane, and appears in dashed line for the next plane. There are also disclination lines perpendicular to all the planes. They are represented on the figure by crossed circles.

\section{Discussion and conclusion.}

There are some common features of all the structures presented. All these structures can be described as a packing of tetrahedra more or less distorted. So the f.c.c. structure is left out of these description since it contains tetrahedra and octahedra. Nevertheless it is perhaps possible to describe the f.c.c. structure with the icosahedral order as reference using disclination lines not going through the atomic positions, but going through the centres and faces of the tetrahedra. In all these structures, the coordination polyhedra are icosahedra, or polyhedra obtained from icosahedra by a disclination procedure using $2 \pi / 5$ disclinations. The b.c.c. structure can also be described like this one with $2 \pi / 5$ and $-2 \pi / 5$ disclinations leading to hexagons and squares on the Voronoï cells of atoms.

Nevertheless in this case the icosahedral order completely disappears and there are no pentagons on a Voronoi cell. With the same restriction as for the b.c.c. structure, this can be extended to the $\omega$ phases (or to an equivalent monoatomic structure of high pressure Ti [12]).

There is another way to present these structures. Suppose that they are defined in a corrugated 3D space (embedded in a space of higher dimension) so that all interatomic distances are equal. In this space there are regions with positive curvature and there are other regions with negative curvature. The present description correspond to the corrugated description if the positive curvature is supposed to be homogeneously dispersed in the whole 3D space, and if the negative curvature is concentrated on disclination lines.

The mean coordination number $\bar{n}$ found in these structures can be related to the value 13.40 of the coordination number obtained by Coxeter [13] for sphere packing. The different values appear in table I. Following Rivier [14] the deviation from the 13.40 value is related to fluctuation in size of the Voronoï cell (decreasing $\bar{n}$ ) or to large deformation relatively to a spherical shape (increasing $\bar{n}$ ). That is verified in this case if we compare the $\bar{n}$ value obtained for Laves phases (13.33) in which there is a big difference in atomic size of the two types of atoms, to the value $\bar{n}=14$ obtained with b.c.c. structure in which all atomic sizes are equal, with a Voronoï cell having a low symmetry (compared to a dodecahedron). In table I the mean number of tetrahedra with a common edge are presented.

The existence of such phases evidently depends on electronic structure [15], but with this description we have a topological explanation of their stability. In terms of a naive description of pair potential energy the existence of such structures can be explained with very flat potential variation near the minimum distance. In this case an increase of the coordination number at the expense of interatomic first distance fluctuations corresponds to a minimum of the energy. 
Table I. - Coordination in metal structures. Notice that the two numbers $\bar{n}$ and $\bar{n}_{\mathrm{t}}$ are related by $\bar{n}\left(6-\bar{n}_{\mathrm{t}}\right)=12$ (from Euler relation). If regular tetrahedra are packed with a common edge there is place for 5.1043 tetrahedra.

\begin{tabular}{|c|c|c|c|c|c|c|c|}
\hline \multirow[b]{2}{*}{ Structure } & \multicolumn{5}{|c|}{$\begin{array}{l}\text { Number of atoms in the cell } \\
\text { with the coordination number }\end{array}$} & \multirow{2}{*}{$\begin{array}{c}\text { Mean } \\
\text { coordination } \\
\text { number } \\
\bar{n}\end{array}$} & \multirow{2}{*}{$\begin{array}{c}\text { Mean value } \\
\text { for the number } \\
\text { of tetrahedra } \\
\text { with a common } \\
\text { edge } \\
\bar{n}_{\mathrm{t}}\end{array}$} \\
\hline & I & II & III & IV & V & & \\
\hline $\begin{array}{l}\beta \text {-tungsten (A15) } \\
\text { Laves phase } \\
\left(\mathrm{Cu}_{2} \mathrm{Mg}\right) \\
\alpha \text {-manganese } \\
\beta \text {-uranium } \\
\text { Body centred cu- } \\
\text { bic }\end{array}$ & $\begin{array}{l}2(12) \\
8(12) \\
2(16) \\
2(12) \\
2(14)\end{array}$ & $\begin{array}{l}6(14) \\
4(16) \\
8(16) \\
4(15)\end{array}$ & $\begin{array}{r}14(14) \\
8(14)\end{array}$ & $\begin{array}{r}24(12) \\
8(12)\end{array}$ & $8(14)$ & $\begin{array}{l}13.50 \\
13.333 \\
13.517 \\
13.466 \\
14\end{array}$ & $\begin{array}{l}5.1111 \\
5.1000 \\
5.1122 \\
5.1089 \\
5.1414\end{array}$ \\
\hline
\end{tabular}

Perhaps also this description can indicate the possibility of an amorphous like behaviour in such metals as for example thermal properties resulting from two-level systems [16, 17]. But that is still an open question.

A similar description can be applied to loose packed structures (Clathrate, Zeolite) with the help of a duality transformation of the close packed structure.

\section{Remarks and acknowledgments.}

This point of view on the structure of alloys was, more or less implicitly, in Frank and Kaspers work and appears very natural if the " curved space " description of the order is taken into account. In parallel to the present work, D. Nelson [18] has just presented a similar approach of the complex alloy structures.

This study has benefited from fruitful discussions with J. Friedel and R. Mosseri.

\section{References}

[1] SAdoc, J. F., J. Non-Cryst. Solids 44 (1981) 1.

[2] COXETER, H. S. M., Regular polytopes (Dover Pub.) 1973.

[3] SADOC, J. F. and Mosseri, R., Philos. Mag. B 45 (1982) 467.

[4] COXETER, H. S. M., Regular Complex polytopes (Cambridge University Press) 1974.

SADOC, J. F. and Mosseri, R., Proceedings of the 1982 Taniguchi Symposium on the Nature of Topological Disorder (Springer-Verlag, to be published).

[5] Frank, F. C. and Kasper, J. S., Acta Cryst. 11 (1958) 184 and 12 (1959) 483.

[6] Morcom, W. R., Worrel, W. L., Sell, H. G. and Kaplan, H. I., Metal. Trans. 5 (1974) 155.

[7] Steward, G. R., Newkirk, L. R. and Valencia, Phys. Rev. B 21 (1980) 5055.

[8] Vieland, L. J. and Wicklund, A. W., Phys. Lett. 49A (1974) 407.

[9] Kimoto, K. and NishidA, I., J. Phys. Soc. Japan 22 (1967) 744.

[10] Oberteuffer, J. A. and Ibers, J. A., Acta Crystal. B 26 (1970) 1499.

[11] The structure of the elements (J. Donohue, J. Wiley ed.) 1974. 
[12] JAMIESON, J. C., Science 140 (1963) 72.

[13] COXETER, H. S. M., Illinois J. Math. 2 (1958) 746.

[14] Rivier, N., J. Physique Colloq. 43 (1982) C9-91.

[15] Turchi, P., Tréglia, G. and Ducastelle, F., to be published in J. Phys. F.

[16] Lou, L. F., Solid State Commun. 19 (1976) 335.

[17] Thomas, N., Arnold, W., Gmelin, E., Guckelsberger, K., Weiss, G. and löhneysen, H. V., J. Physique Colloq. 41 (1980) C8-751.

[18] Nelson, D., Phys. Rev. Lett. 13 (1983) 983. 